\title{
REVISIÓN
}

\section{La cromatografía líquida de alta eficacia en la separación de triglicéridos de grasas animales complejas}

\author{
Por Javier S. Perona ${ }^{1}$, Valentina Ruiz-Gutiérrez ${ }^{1}$ y Luis J.R. Barrón ${ }^{2}$ \\ 1 Instituto de la Grasa, (CSIC). Avda. Padre García Tejero, 4. 41012 - Sevilla. \\ 2 Tecnología de Alimentos. Universidad del País Vasco/Euskal Herriko Unibertsitatea. \\ Paseo de la Universidad, 7. 01006 - Vitoria-Gasteiz.
}

\section{RESUMEN}

La cromatografía líquida de alta eficacia en la separación de triglicéridos de grasas animales complejas.

Los triglicéridos son una de las fracciones lipídicas más abundante y al mismo tiempo más compleja de la naturaleza. Por esta razón, es esencial la determinación de sus especies moleculares, así como conocer la posición estereoespecífica en que se sitúan los ácidos grasos que los componen, para caracterizar las grasas animales y los aceites vegetales. La técnica más comúnmente empleada con este fin es la cromatografía líquida de alta eficacia (HPLC), en general utilizando columnas de fase reversa. En el presente artículo se describen las condiciones operativas que actualmente se emplean para alcanzar la óptima resolución de picos cromatográficos de triglicéridos, así como la situación actual del conocimiento en materia de separación de especies moleculares de mezclas de triglicéridos complejas, tales como la grasa láctea, e aceite de pescado y la grasa hepática, que son de importancia en el sector industrial y en la nutrición humana.

PALABRAS-CLAVE: Aceite de pescado - Cromatografía líquida de alta eficacia - Grasa de leche - Grasa hepática - Revisión (artículo) - Triglicérido.

\section{SUMMARY}

High-performance liquid chromatography in the separation of triglycerides from complex animal fats.

Triglycerides are at the same time, one of the most abundant and complex lipid fraction in the nature. For this reason, it is esential to determine their molecular species composition, as well as to know the stereospecific position in which the fatty acids are located, in order to characterize animal fats and vegetable oils. The most commonly employed technique for this objective is high-performance liquid chromatography, in general using reversed phase columns. In the present article we describe the operative conditions employed nowadays in order to achieve the optimum resolution for the triglyceride chromatographic peaks, and also the present situation of the knowledge in the separation of the molecular species of complex mixtures of triglycerides, such as milk fat, fish oil and hepatic fat, which are of importance in the industrial sector and human nutrition.

KEY-WORDS: Fish oil - Hepatic fat - High-performance liquid chromatography - Milk fat - Review (paper) - Triglyceride.

\section{INTRODUCCIÓN}

La caracterización de los lípidos, procedentes de grasas animales o aceites vegetales, ha sido una ardua tarea, marcada por la determinación de la composición de los ácidos grasos que los forman. Sin embargo, en la actualidad se puede afirmar que la caracterización de una grasa o un aceite no está completa sin la determinación de las especies moleculares de las clases de lípidos que la componen. De este modo es posible conocer de qué forma se distribuyen los ácidos grasos dentro de cada molécula lipídica. En cualquier tipo de grasa, es importante el estudio de las especies moleculares de sus triglicéridos (TGs), puesto que se trata de la fracción lipídica más abundante en la naturaleza, además de la más compleja (Berqvist y Kaufmann, 1993). Bajo este prisma, cada día se atribuye una mayor importancia al análisis de TGs.

Con anterioridad a su análisis, los TGs deben ser aislados del resto de fracciones lipídicas. Si bien esto no es necesario en el caso de los aceites vegetales, en los que los TGs se constituyen en fracción mayoritaria (alrededor del 95\%), sí resulta un problema en el caso de las grasas animales, en cuyo caso, los lípidos polares se encuentran en mayor cantidad, causando interferencias en el análisis. En la actualidad, con este fin se viene utilizando la cromatografía en capa fina (TLC), utilizando un sistema de elución compuesto por mezclas hexano/éter etílico en distintas proporciones (Ruiz-Gutiérrez et al., 1993, Perona et al., 1998a, y Perona et al., 1998 b). Recientemente se ha propuesto la extracción en fase sólida (Solid Phase Extraction, SPE) como un método más rápido e igualmente eficaz (Kaluzny et al., 1998, Pacheco et al., 1998).

Una vez aislados los TGs, con el fin de separar sus especies moleculares, se han empleado, con mayor o menor éxito, diversas técnicas cromatográficas. La cromatografía en capa fina con iones de plata (Ag-TLC) separa los TGs en diversas fracciones, de 
las cuales posteriormente se estudia su composición en ácidos grasos mediante cromatografía de gases. Esta última técnica también se emplea para la determinación de TGs usando columnas capilares de sílice rellenadas con fenilmetilsilicona polarizable a alta temperatura. Hoy en día se sigue empleando para la determinación de algunas grasas y aceites sencillos poco insaturados, con resultados, en ocasiones, comparables a los de la cromatografía líquida (CareIli y Cert, 1993 y Perona et al., 1998b).

Hace casi un cuarto de siglo, Pei et al., (1975) publicaron el primer trabajo en el que se proponía un método de HPLC para la separación de TGs de cadena media en una columna de fase reversa. Desde ese momento, otros investigadores han continuado la mejora de los métodos para la separación de TGs. El primer fraccionamiento de una grasa natural se consiguió dos años después (1977) y por dos autores por separado: Wada et al., y Plattner et al. Los primeros, además, establecieron un parámetro, denominado Número de Partición (NP; NP $=N C-2$ $\mathrm{ND}$, donde $\mathrm{NC}$ es el número de carbonos totales del TG y ND es el número de dobles enlaces situados en los ácidos grasos que componen la molécula de TG), para caracterizar molecularmente los TGs. Estos autores descubrieron que los TGs eran eluídos en la columna de fase reversa en orden creciente de NP. Hoy en día la cromatografía líquida de alta eficacia (highperformance liquid chromatography, HPLC) es la técnica más empleada y la que mejores resultados ofrece, para el análisis de las especies moleculares de TGs, que son separadas en base a su longitud de cadena, su número de insaturaciones, su polaridad y su configuración, lo que permite una determinación precisa de la composición triglicerídica de una grasa.

No obstante, considerables inconvenientes han tenido que ser sorteados antes de lograr la eficacia que el sistema de HPLC tiene hoy en día. El primer problema surgió en la separación de lo que se denominó «pares críticos", moléculas que poseían un comportamiento similar cuando eran eluídas en una columna de fase reversa, a pesar de tener diferente número de carbonos 0 diferente número de dobles enlaces. Además, hasta la aparición del primer método que contaba con una fase móvil provista de un sistema de elución en gradiente, la separación de TGs con grandes diferencias de número de carbonos resultaba muy complicada. Hoy en día se emplean gradientes compuestos por diferentes rampas y se intercalan zonas de elución isocrática y zonas de elución en gradiente. Incluso es posible ir modificando el contenido de la fase móvil en uno u otro componente de manera exponencial. De esta forma, es posible resolver en un mismo cromatograma TGs de distinto NC, así como muchos de los considerados pares críticos.

Otra de las dificultades que hubo que superar, era la detección de los analitos a la salida de la columna. De entre los diversos detectores que se han venido empleando, ha sido el detector de difusión de luz evaporativo (evaporative light-scattering detector, ELSD), el que mejores resultados ha ofrecido, si bien, el acoplamiento del HPLC a un espectrómetro de masas ofrece una gran ayuda en la identificación de las especies moleculares. Este último es uno de los mayores problemas que la técnica de HPLC para el análisis de TGs aún no ha resuelto.

Finalmente, la determinación de la composición triglicerídica de las grasas y aceites vegetales ha quedado resuelta satisfactoriamente, aun cuando quedan algunos pares críticos por separar (Barrón y Santa-María, 1989). Sin embargo, algunas grasas animales, presentan un número mucho mayor de pares críticos, y picos cromatográficos por identificar. $A$ medida que el número de ácidos grasos constituyentes de una grasa aumenta, el número de posibles combinaciones entre ellos para formar TGs se eleva enormemente, con el consiguiente aumento del número de pares críticos y de las dificultades en su separación e identificación. Pero además, cuando existe una gran variedad de longitudes de cadena y de insaturaciones entre los ácidos grasos, da lugar a mezclas tan complejas de TGs que no pueden ser completamente separadas mediante el concurso de una única técnica cromatográfica. Ambas situaciones tienen lugar en las grasas lácteas y los aceites de pescado.

En el presente trabajo se discuten las condiciones cromatográficas que se han propuesto para el análisis de mezclas complejas de TGs por HPLC. Además, se describe el estado actual de conocimientos en el campo de la separación de TGs de algunas de las grasas animales más complejas y de importancia para el sector industrial y la nutrición humana, como la grasa láctea, el aceite de pescado y la grasa de hígado.

\section{CONDICIONES OPERATIVAS ÓPTIMAS PARA EL ANÁLISIS DE TRIGLICÉRIDOS POR HPLC}

\section{Fase Móvil}

La elección de la fase móvil es uno de los factores que más influyen en el análisis cromatográfico de TGs. Desde los primeros experimentos, se estudió cuál podía ser la mejor combinación de disolventes para la resolución de las especies moleculares de TGs cuando se empleaban columnas de fase reversa (Platanar et al., (1977). Pals (1983), concluyó que el acetonitrilo era el más idóneo para ser utilizado como disolvente principal, puesto que con él se alcanzaba la mejor separación de pares críticos. Recientemente, Hirano y Takahasi (1996) han establecido las propiedades de la mezcla de elución que deben equilibrarse para lograr la óptima eficacia de la columna, proponiendo que la mezcla debe disolver 
perfectamente los TGs, pero además deben ser de bajo peso molecular y viscosidad.

Con el fin de mejorar la solubilidad de los compuestos, así como proporcionar cambios en su polaridad, y por lo tanto en la selectividad de los picos cromatográficos, se añade al disolvente principal, un disolvente secundario, denominado modificador orgánico. Dada la relativa apolaridad de los TGs, un aumento en la polaridad de la fase móvil, se refleja invariablemente en un incremento del tiempo de retención y mejora la resolución de picos, incluyendo los pares críticos. El modificador orgánico más comúnmente empleado para la resolución de especies moleculares de TG es la acetona. Dado que la acetona absorbe la luz ultravioleta (UV) en las mismas longitudes de onda que los TGs (200$237 \mathrm{~nm}$ ) (Shukla et al., 1983), el detector de UV se utiliza cada vez con menor frecuencia.

Durante mucho tiempo, el análisis de TGs por HPLC se llevó a cabo con elución isocrática (Goiffon et al., 1981, Lozano, 1983). Sin embargo, para la separación de TGs de grasas complejas, se hace necesaria la utilización de gradientes, que implican la variación del porcentaje de modificador orgánico en la mezcla. De este modo, es posible resolver mezclas complejas de TGs, como las del aceite de pescado, que contiene ácidos grasos poliinsaturados de cadena larga o como las de la grasa de la leche con un amplio rango de valores de NP (Berqvist y Kaufmann, 1993, Nájera et al., 1998). Los sistemas de gradiente pueden ser lineales o no. Generalmente los no-lineales son los que alcanzan mejores separaciones de pares críticos.

\section{Disolvente de la Muestra}

El disolvente de la muestra adquiere gran importancia cuando es necesario solubilizar TGs con grandes diferencias de polaridad. Además, el disolvente debe permitir un correcto contacto entre el soluto y la fase estacionaria. Se han propuesto como disolventes aceptables el cloroformo, el hexano y la propia fase móvil, rechazándose otros como la acetona porque no es adecuada para TGs saturados de alto peso molecular (Tsimidou y Macrae, 1985).

\section{Fase Estacionaria}

Las fases estacionarias de fase reversa o inversa, compuestas por partículas de octadecilsilano (ODS), la mayoría con forma esférica y soportada en empaquetamientos de sílice, han demostrado proporcionar la mejor selectividad para TGs, sobre todo aquéllas de menor tamaño de partícula, como son las de $3 \mu \mathrm{m}$. Generalmente la longitud de cadena uti- lizada no supera los $30 \mathrm{~cm}$, ya que se ve limitada por las altas presiones necesarias y por los elevados tiempos de retención resultantes (Ruiz-Gutiérrez y Barrón, 1995a).

\section{Detectores}

Prácticamente todos los detectores posibles han sido probados y empleados para el análisis de TGs. Cuando se utiliza elución isocrática, los detectores de índice de refracción (IR) ofrecen resultados satisfactorios (Goiffon et al., 1981, Lozano, 1983), pero cuando es necesario aplicar sistemas de gradiente, dejan de tener utilidad debido a la desmesurada deriva que producen (Ruiz-Gutiérrez y Barrón, 1995a). Los detectores de ultravioleta (UV), son compatibles con gradientes, pero no con algunos de los disolventes más utilizados para separar TGs, puesto que absorben en la misma región del espectro que éstos (200-230 nm, enlace éster). Además los TGs tienen coeficientes de extinción molar no uniformes, y consecuentemente es necesario calcular sus factores de respuesta mediante patrones para un análisis cuantitativo (Singleton et al., 1984, Nurmela y Satama, 1988, Barrón et al., 1989). Esta misma deficiencia fue encontrada también en los detectores de ionización de llama (FID), además de respuestas no lineales al inyectar cantidades por debajo de $5 \mu \mathrm{g}$ (Nurmela y Satama, 1988).

La introducción del detector de difusión de luz evaporativo (ELSD) ha propiciado un gran avance en la detección de TGs. Dado que su respuesta es simplemente proporcional a la masa del analito, este detector no está limitado por las características espectrales de los compuestos. Además, ya que los disolventes son eliminados por evaporación antes de la detección, no provocan deriva de la línea base (Charlesworth, 1978, Guiochon et al., 1988, Riguezza y Guiochon, 1988).

A pesar de su utilidad, los detectores descritos tienen la desventaja de la identificación de los picos cromatográficos, que se evita utilizando como detector un espectrómetro de masas. Mediante la combinación de un sistema de HPLC y un espectrómetro de masas de ionización química a presión atmosférica se pueden obtener datos relativos al peso molecular de los TGs, lo que facilita enormemente su identificación.

\section{Identificación de Especies Moleculares}

Dada la dificultad que existe aún hoy en día para disponer de un equipo integrado de HPLC-espectrómetro de masas, se han concebido otros sistemas para la predicción de la identidad de los picos cro- 
matográficos. La relación lineal entre el factor de capacidad $\left(k^{\prime}\right)$ y los valores de NP de los TGs fue establecida por primera vez por Wada et al., (1977). Posteriormente Herslöf et al., (1979), estimaron teóricamente el Número Equivalente de Carbonos (NEC) para TGs insaturados, en base a sus tiempos de retención relativos, a partir de una relación lineal experimental entre esos tiempos de retención relativos y el NC. EI NEC de cada TG en la muestra se define como el NEC del TG saturado hipotético, que tuviera el mismo tiempo de retención. El NEC es análogo al NP (NEC=NC - a'ND), con la diferencia de que en este caso el valor de a' depende de cada sistema cromatográfico. Sin embargo, a' toma generalmente valores cercanos a I2I y cuando $a^{\prime}=2$ entonces NEC=NP. Takahashi et al., (1985) calculó el valor de a' a partir de la relación entre log k', NC y ND $\left(\log a^{\prime}=q+b^{\prime *} N C+c^{\prime *} N D\right)$, donde el valor de a' es el cociente entre las constantes $b^{\prime} y c^{\prime}$.

En 1992, Hierro et al., señalaron que el comportamiento de los triglicéridos en HPLC en fase inversa no acuosa dependía no sólo del NC y ND, sino también del número de ácidos grasos insaturados en la molécula (NAI), ya que triglicéridos con igual valor de NEC eluían en orden creciente de número de ácidos grasos saturados constituyentes. De acuerdo a estos resultados, Nájera et al., (1998) han redefinido el NEC como una función que depende del NC, ND y NAl, como NEC = NC - 2. ND - 0.2. NAI.

El proceso de predicción de los TGs se hace extremadamente complicado a medida que aumenta el número de ácidos grasos constituyentes, ya que el número posible de TGs se eleva enormemente. Por esta razón, y como una segunda parte del proceso de predicción, algunos autores han propuesto la aplicación de las ecuaciones desarrolladas por Takahashi et al., (1985). Estos autores desarrollaron un modelo matricial cuyas variables eran NC y ND de cada ácido graso que esterifica la molécula de glicerol.

\section{Análisis Estereoespecífico}

Para la completa caracterización de una grasa es necesario no sólo conocer cuáles son los ácidos grasos que componen sus TGs sino también saber en qué posición estereoespecífica se encuentran localizados dentro de la molécula de glicerol, ya que de ello dependen algunas de sus propiedades fisicoquímicas. Sin embargo, el análisis estereoespecífico resulta particularmente dificultoso, puesto que las moléculas que han de separarse entre sí son extremadamente parecidas en relación a sus propiedades fisicoquímicas.

La metodología de análisis por HPLC, que permite conocer la distribución estereoespecífica de los ácidos grasos, utiliza mono y diglicéridos como sustrato de análisis. Estos glicéridos parciales se obtienen tras la hidrólisis de los TGs, que generalmente se realiza mediante la reacción de Grignard (Yurkowsky y Brockerhoff, 1966). Los productos obtenidos son posteriormente analizados por HPLC utilizando columnas de fase normal o reversa. La combinación de este análisis junto con la composición en ácidos grasos obtenida por cromatografía de gases permite el cálculo de las posiciones estereoespecíficas de los TGs de una determinada grasa (Christie, 1987). En el primer caso, los 1,2 y 1,3 diglicéridos se derivatizan a (S)-(+)-1-(1-naftil)etil uretanos (Christie, 1987), mientras que en fase reversa se derivatizan a 3,5-dinitrofenil uretanos (DNFU) (Semporé y Bezard, 1991).

Finalmente, mayor éxito ha tenido la utilización del HPLC en fase quiral. Esta fase estacionaria presenta moléculas quirales adheridas a la sílice, lo que evita tener que utilizar derivados diasteroisoméricos. De todos modos se han encontrado importantes desventajas, como altos tiempos de retención y pobre resolución. Este método separa los diglicéridos 1,2 de los 1,3, que posteriormente deben ser analizados mediante cromatografía de gases para determinar su composición en ácidos grasos (Itabashi y Takagi, 1986; Ruiz-Gutiérrez y Barrón, 1995b).

\section{COMPOSICIÓN TRIGLICERÍDICA DE LA GRASA LACTEA}

Los constituyentes mayoritarios de la grasa láctea son los TGs, con un $98.7 \%$ del total de lípidos. Otros componentes son los fosfolípidos $(0.8 \%)$, principalmente fosfatidil-etanolamina, fosfatidil-colina y esfingomielina, además de esteroles, ácidos grasos libres, carotenoides y vitaminas liposolubles, que se presentan en conjunto en cantidades inferiores al 0.5\% (Alais, 1985; Christie, 1987; Walstra y Jenness, 1987; Jensen et al., 1991).

Los trabajos sobre la composición de la grasa láctea se han centrado en el estudio de los ácidos grasos y en la descripción de las clases de TGs según el peso molecular, grado de insaturación y número total de carbonos de sus ácidos grasos constituyentes. La mayor parte de los estudios se han dedicado a las leches de vaca, oveja o cabra.

Según Walstra y Jenness (1987) la grasa láctea contiene aproximadamente 64 ácidos grasos de longitudes de cadena comprendidas entre $\mathrm{C}_{4}$ y $\mathrm{C}_{26}$, de los cuales cerca de la tercera parte se encuentran en proporciones individuales menores del $0.1 \%$. En general, los ácidos grasos saturados representan aproximadamente el $62 \%$ del total de ácidos de la grasa láctea, de los cuales solamente un $2 \%$ presenta un número impar de átomos de carbono y cerca de un $1 \%$ son de cadena ramificada. Los ácidos grasos insaturados representan el $38 \%$ del total de ácidos, siendo mayoritarios los ácidos monoinsaturados (34\%) frente a los poliinsaturados (4\%) (Christie, 1983). 
La grasa láctea presenta una composición mayoritaria en los ácidos grasos palmítico $\left(\mathrm{C}_{16: 0}\right)$ y oleico $\left(\mathrm{C}_{18: 1}, \mathrm{n}-9\right)$, con porcentajes individuales entre el 20 y $30 \%$, siendo ligeramente superior el contenido de ácido palmítico. Los ácidos grasos esteárico $\left(\mathrm{C}_{18: 0}\right)$ y mirístico $\left(\mathrm{C}_{14: 0}\right)$ presentan porcentajes entre el 10 y $15 \%$, mientras que el ácido laúrico $\left(\mathrm{C}_{12: 0}\right)$ se encuentra cercano al $5 \%$. Los ácidos grasos de cadena corta, butírico $\left(\mathrm{C}_{4: 0}\right)$, caproico $\left(\mathrm{C}_{6: 0}\right)$, caprílico $\left(\mathrm{C}_{8: 0}\right)$ y cáprico $\left(\mathrm{C}_{10: 0}\right)$, característicos de la grasa láctea, se encuentran en porcentajes individuales entre el 2 y $10 \%$. Los ácidos poliinsaturados linoleico $\left(C_{18: 2}\right.$, $n-6)$ y linolénico $\left(C_{18: 3}, n-3\right)$ se presentan en porcentajes individuales generalmente inferiores al $2 \%$ (Galvano y Serra, 1984; Anifantakis, 1986; Ramos y Juárez, 1986; González de Llano y Ramos, 1989; Lomascolo et al., 1994; Jandal, 1996). Por otra parte, se han encontrado ácidos trans-insaturados, fundamentalmente ácido vaccénico $\left(\mathrm{C}_{18: 1}, \mathrm{n}-7\right)$ y octadecen-16-oico $\left(\mathrm{C}_{18: 1}, \mathrm{n}-2\right)$, con porcentajes entre el 2 y el $11 \%$ (Deman y Deman, 1983; Zegarska, 1988; Wolff, 1995).

La composición en TGs individuales de la grasa láctea presenta una enorme complejidad. Considerando los ácidos grasos presentes en la grasa láctea en proporciones superiores al 1\%, serían más de 1300 los TGs posibles (Walstra y Jenness, 1987). Probablemente debido a las dificultades analíticas que entraña el estudio de esta compleja mezcla, en la bibliografía se han encontrado muy pocos trabajos que describan la composición molecular individual de los TGs de la grasa láctea.

Según el peso molecular, los TGs mayoritarios de la grasa láctea corresponden a la fracción de peso molecular alto (PMA) (53\% del total de TGs), seguidos de los de peso molecular bajo (PMB) $(28 \%)$ y de los de peso molecular medio (PMM) (19\%), determinados a partir de su movilidad en cromatografía de capa fina en gel de sílice. (Arumughan y Narayanan, 1982).

Según el grado de insaturación, los TGs mayoritarios de la grasa láctea corresponden a los cis-monoenos $(33 \%)$, seguidos de los trisaturados $(30 \%)$, dienos (21\%), trans-monoenos $(9 \%)$ y polienos $(7 \%)$ (Arumughan y Narayanan, 1982; Parodi, 1982). En la fracción de TGs trisaturados los ácidos mayoritarios son el palmítico $\left(\mathrm{C}_{16: 0}\right)(32 \%)$, mirístico $\left(\mathrm{C}_{14: 0}\right)(18 \%)$ y esteárico $\left(C_{18: 0}\right)(12 \%)$. En general, en las fracciones de dienos y polienos, los ácidos insaturados de cadena larga, en particular el ácido oleico $\left(\mathrm{C}_{18: 1}, \mathrm{n}-9\right)$ se combinan con ácidos de cadena corta o media (Barbano y Sherbon, 1979; Arumughan y Narayanan, 1982; Banks et al., 1987). Según Barrón et al., (1990), las moléculas trisaturadas en la grasa láctea representan un $43 \%$ del número total de TGs, las monoinsaturadas un $31 \%$, las diinsaturadas un $12 \%$, y finalmente las poliinsaturadas un $14 \%$. Por otra parte, según Ruiz-Sala et al., (1996), el 44\% del total de TGs de la grasa láctea son moléculas saturadas, el $24 \%$ monoinsaturadas y el $32 \%$ poliinsaturadas.

Según el número total de carbonos (NC), los TGs de la grasa láctea están comprendidos entre NC 22 y 54, siempre con número par de átomos de carbono, siendo los mayoritarios los de NC medio, 34, 36, 38 y 40, y NC largo, 50 y 52 (Parodi, 1980; Caboni et al., 1982; Marjanovic et al., 1984; Kuksis et al., 1986; Banks et al., 1987; Lund, 1988; Gresti et al., 1993; Molkentin y Precht, 1994; Precht y Frede, 1994; Contarini y Toppino, 1995).

De acuerdo con el Número de Partición (NP), Barrón et al., (1990) fraccionaron los TGs de la grasa láctea de vaca, oveja y cabra en 15 clases distintas comprendidas entre NP 22 y 50, siempre con un valor par de NP, resultando mayoritarios los TGs correspondientes a la fracción de NP 46. Sin embargo, estos resultados difieren de los encontrados por otros investigadores, para los que los TGs mayoritarios de la leche de vaca correspondían a los grupos de NP 38 y 48 (Frede y Thiele, 1987), NP 36 y 48 (Weber et al., 1988b) y NP 34 y 36 (Hinrichs et al., 1992). Ruiz-Sala et al., (1996) encontraron que los TGs de la grasa de leche de vaca, oveja y cabra presentaban valores de NP entre 24 y 54, de los que resultaban ser mayoritarios los de NP 36 y 46 . Por otra parte, Nájera et al., (1998) han fraccionado los TGs de la grasa de queso de oveja en 32 clases distintas comprendidas entre los NP 22 y 53 (Figura 1). Los TGs fueron fundamentalmente de número de partición par, entre los que destacaron los grupos de NP 34, 36 y 38 .

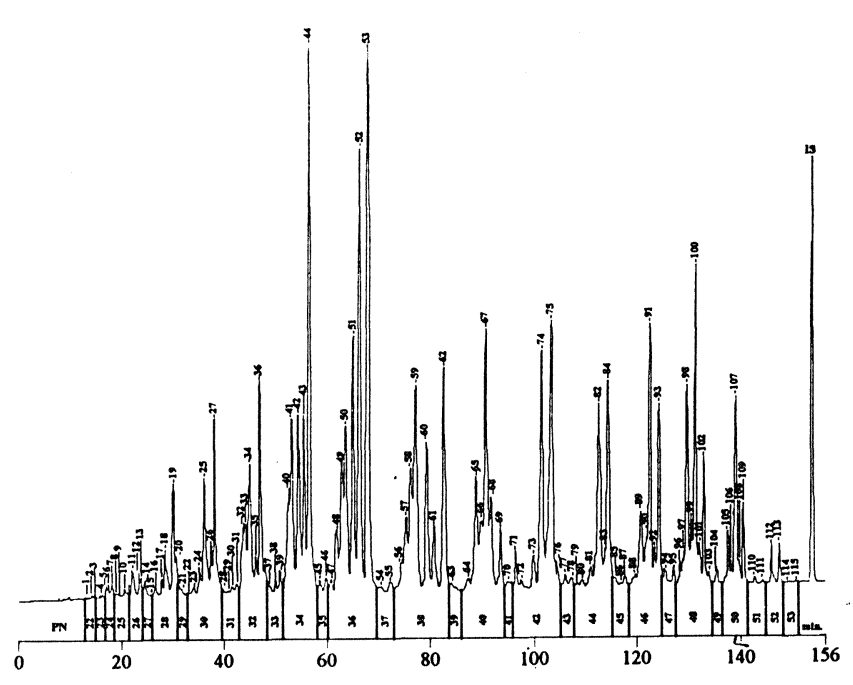

Figura 1

Cromatograma de triglicéridos de la grasa de queso Idiazabal mediante HPLC. Separación llevada a cabo a $30^{\circ} \mathrm{C}$, empleando dos columnas Nucleosil 120C-18 (3 $\mu \mathrm{m}, 200 \times 4.6 \mathrm{~mm})$ dispuestas en serie. Gradiente de acetona en acetonitrilo 0-35\% de acetona en 50 min., isocrático 20 min, aumentando a $80 \%$ de acetona en $75 \mathrm{~min}$ e isocrático los últimos $10 \mathrm{~min}$. Disolvente de inyección $n$-hexano, $10 \mu$ l.

$\mathrm{PN}=$ número de partición; IS = patrón interno (triestearina). (Nájera et al., Chromtogr. 1998, 47:576-579). 
Otros autores han fraccionado los TGs en clases según el número equivalente de carbonos (NEC). Ruiz-Sala et al., (1996) obtuvieron en las leches de vaca, oveja y cabra 111 fracciones comprendidas entre los valores de NEC 24.0 y 54.0. Según estos autores, la leche de oveja presentó un porcentaje mayor de TGs de NEC $\leq 34.0$, con valores del $18 \%$, frente a los correspondientes a las leches de vaca y cabra, 10 y 15\%, respectivamente. Los porcentajes de TGs con NEC entre 36 y 40 fueron del mismo orden en las leches de las tres especies, 33, 25 y 31\%, respectivamente para la de oveja, vaca y cabra, y finalmente la leche de vaca presentó el mayor porcentaje de TGs de NEC > 40, con valores del $64 \%$ frente al 54 y $49 \%$ para la de cabra y oveja, respectivamente.

Weber et al., (1988b) identificaron 122 TGs en la grasa láctea de vaca resultando mayoritarios los TGs de NP 36, butiroil-dipalmitil-glicerol (BPP), butiroil-miristoil-estearil-glicerol (BMS), butiroil-palmitoiloleil-glicerol (BPO) y caproil-miristoil-palmitil- glicerol (CMP), y los TGs de NP 48, dipalmitoil-oleil-glicerol (PPO), miristoil-estearoil-oleil-glicerol (MSO) y palmitoil-dioleil-glicerol (POO). Barron et al., (1990) identificaron un total de 116 TGs individuales en la grasa láctea de vaca, oveja y cabra. Los TGs identificados, pertenecientes a fracciones mayoritarias de NP, fueron miristoil-dioleil-glicerol en la fracción de NP 46, miristoil-oleoil-linoleil-glicerol (MOL) y lauroil-dipalmitilglicerol (LaPP) en la fracción de NP 44 y butiroil-miristoil-oleil-glicerol (BMO) y butiroil-miristoil-palmitil- glicerol (BMP) en la fracción de NP 34.

Por otra parte, Gresti et al., (1993), identificaron 404 TGs individuales en la grasa láctea de vaca, de los cuales resultaron ser mayoritarios butiroil-palmitoil-oleil-glicerol (BPO), butiroil-dipalmitil-glicerol (BPP) y butiroil-miristoil-palmitil-glicerol (BMP). Unicamente 22 fueron los TGs superiores al $1 \%$ en peso, representando el $42.7 \%$ del total. Spanos et al., (1995) identificaron, mediante cromatografía líquida y espectrometría de masas, como TGs principales de la grasa láctea de vaca, butiroil-dipalmitil-glicerol (BPP), dipalmitoil-oleil-glicerol (PPO), butiroil-palmitoiloleil-glicerol (BPO) y butiroil-miristoil-estearil-glicerol (BMS). Ruiz-Sala et al., (1996) identificaron mediante la combinación de cromatografía de líquidos y de gases, 181 TGs en la grasa de leche de vaca, oveja y cabra, de los que resultaron ser mayoritarios caproilmiristoil-palmitil-glicerol (CMP), dicaprioil-palmitil-glicerol (CCP), butiroil-dipalmitil-glicerol (BPP) y butiroil-miristoil-estearil-glicerol (BMS), pertenecientes a la fracción de NP 36, y dipalmitoil-linoleil-glicerol (PPL) miristoil-estearoil-linoleil-glicerol (MSL), miristoilpalmitoil-oleil-glicerol (MPO), miristoil-palmitoil-vaccenilglicerol (MPV) y lauroil-estearil-oleil-glicerol (LSO), pertenecientes a la fracción de NP 46. Recientemente, Nájera et al., (1998) han identificado como moléculas mayoritarias de la grasa de queso de oveja TGs que contenían en su composición ácido butírico, butiroildipalmitil-glicerol (BPP), butiroil-miristoil-palmitil-glicerol (BMP) y butiroil-palmitoil-oleil-glicerol (BPO) (Figura 1).

Todos los estudios comentados anteriormente no tuvieron en cuenta la composición estereoespecífica de los TGs. En este sentido, se han llevado a cabo numerosos estudios, principalmente en leche de vaca, para la determinación de la distribución posicional de los ácidos grasos en la molécula del glicerol. Todos los estudios muestran que la distribución de los ácidos grasos no es al azar, y que presenta un alto grado de asimetría.

En general, los ácidos grasos butírico $\left(\mathrm{C}_{4: 0}\right)$ y caproico $\left(\mathrm{C}_{6: 0}\right)$ esterifican la posición $s n-3$ del glicerol; ocurre lo mismo pero en menor proporción con los ácidos grasos comprendidos entre el ácido caprílico $\left(\mathrm{C}_{8: 0}\right)$ y decenoico $\left(\mathrm{C}_{10: 1}\right)$. Los ácidos grasos desde el laúrico $\left(\mathrm{C}_{12: 0}\right)$ al pentadecanoico $\left(\mathrm{C}_{15: 0}\right)$ se sitúan preferentemente en la posición sn-2; el ácido palmítico $\left(\mathrm{C}_{16: 0}\right)$ se distribuye entre las posiciones $s n-1$ y $s n-2$; el ácido esteárico $\left(\mathrm{C}_{18: 0}\right)$ ocupa preferentemente la posición sn-1 y los ácidos poliinsaturados de cadena larga se distribuyen entre las tres posiciones de la molécula del glicerol, predominando en la sn-3. Teniendo en cuenta la distribución estereoespecífica de todos los ácidos grasos en la molécula de glicerol, en las posiciones $s n-1$ y $s n-2$ se encuentra en mayor porcentaje el ácido palmítico $\left(\mathrm{C}_{16: 0}\right)$, mientras que en la posición sn-3 predomina el ácido oleico $\left(C_{18: 1}, n-9\right)$ (Blank y Privett, 1964; Christie y Clapperton, 1982; Parodi, 1983; Gresti et al., 1993).

Parodi (1982) encontró en la leche de vaca que la distribución posicional de los ácidos en los TGs cismonoenos y trans-monoenos era prácticamente similar en las fracciones de PMA, PMM y PMB, mientras que en el resto de los TGs insaturados, la distribución posicional variaba según el peso molecular de los mismos. En este sentido, Itabashi et al., (1993) determinaron posteriormente la presencia de los ácidos acético $\left(\mathrm{C}_{2: 0}\right)$ y butírico $\left(\mathrm{C}_{4: 0}\right)$ en la posición $s n-3$ de los TGs de la grasa de leche de vaca.

La composición de la grasa láctea presenta variaciones importantes según factores genéticos, fisiológicos, ambientales y de manejo del ganado, entre los que cabe señalar la raza, la edad, el peso, el período de lactación, la salud del animal, la época del año, la alimentación, el uso de hormonas y medicamentos, técnicas de ordeño (Galvano et al., 1982; Hawke y Taylor, 1983; Cullinane et al., 1984; Chazal y Chilliard, 1986; Klobasa y Farries, 1986; Thompson, 1988; Faulkner y Pollock, 1989; Gibson, 1991; Beaulieu y Palmquist, 1995; Bencini y Pulina, 1997; Pellegrini et al., 1997). De los factores anteriormente mencionados, la época del año es uno de los más importantes.

Las diferencias encontradas en la composición en ácidos grasos de la grasa láctea según la época 
del año son consecuencia de diferencias en las proporciones entre los ácidos saturados e insaturados. En general, se han observado mayores contenidos de ácido esteárico $\left(C_{18: 0}\right)$, oleico $\left(C_{18: 1}, n-9\right)$ y vaccénico $\left(C_{18: 1}, n-7\right)$ durante los meses de verano, y menores contenidos en la época de invierno, mientras que ocurre lo contrario para el ácido palmítico $\left(\mathrm{C}_{16: 0}\right)$ y mirístico $\left(\mathrm{C}_{14: 0}\right)$ (Parodi, 1970; Cullinane et al., 1984; Frede y Thiele, 1987; Zegarska, 1988; Baer, 1991; Hinrichs et al., 1992; Palmquist et al., 1993).

Jensen y Clark (1988) han propuesto que los cambios estacionales en la composición de los ácidos grasos de la leche son debidos a efectos nutricionales relacionados con la alimentación de los animales. Así, Parodi (1970) encontró que el bajo contenido en ácidos $\mathrm{C}_{18}$ en los meses de invierno era debido a una dieta nutritiva no óptima, lo que provocaba que la síntesis de la grasa láctea dependiera en gran medida de las reservas corporales. Cuando los animales eran alimentados con grasas ricas en ácidos grasos insaturados de cadena larga, como es el caso del pasto rico en ácidos linoleico $\left(C_{18: 2}, n-6\right)$ y linolénico $\left(C_{18: 2}, n-3\right)$, la leche contenía cantidades importantes de ácido esteárico $\left(\mathrm{C}_{18: 0}\right)$ y oleico $\left(\mathrm{C}_{18: 1}\right.$, $n-9)$, debido a la hidrogenación del ácido linoleico en el rumen, lo que además profería a algunas moléculas configuración trans. El contenido en ácidos grasos de cadena media y corta decrecía porque se inhíbe la síntesis "de novo» al reducirse la relación acetato/propiónico, y por lo tanto la cantidad de precursores de acetato (Christie, 1980; Banks et al., 1983; Zegarska, 1988).

Juárez y Ramos (1986) observaron que al aumentar el aporte energético de la ración, el contenido de los ácidos esteárico $C_{18: 0}$ y oleico $\left(C_{18: 1}, n-9\right)$ descendía, tendiendo a aumentar el contenido de los ácidos del caprílico $\left(\mathrm{C}_{8: 0}\right)$ al palmítico $\left(\mathrm{C}_{16: 0}\right)$, hecho justificado ya que con las dietas de alta energía, la cantidad de ácido acético disponible en el rumen es mayor.

Zegarska y Jaworski (1981) describieron la variación en la composición en TGs en la grasa láctea según la época del año. Observaron que según avanzaba la época de lactación iban disminuyendo los TGs de cadena corta $\left(\mathrm{C}_{26}-\mathrm{C}_{32}\right)$ y de cadena media $\left(\mathrm{C}_{34}-\mathrm{C}_{42}\right)$, ocurriéndoles lo contrario a los de cadena larga $\left(\mathrm{C}_{44}-\mathrm{C}_{54}\right)$, que presentaban mayores contenidos al final de la época de lactación. Así en el período de invierno, cuando la alimentación era a base de concentrados, la leche contenía más TGs de cadena media $\left(\mathrm{C}_{34}-\mathrm{C}_{42}\right)$, mientras que en el período de verano, cuando se alimentaban a base de pasto la leche contenía mayor número de TGs con $\mathrm{C}_{50}, \mathrm{C}_{52} \mathrm{y}$ $\mathrm{C}_{54}$.

Resultados similares fueron obtenidos posteriomente por otros autores. Frede y Thiele (1987) observaron en la grasa de la leche de vaca de verano mayores proporciones de los TGs con NP 44, 46, 48 y 50 que en la leche de invierno, ocurriendo lo contrario con los TGs de NP 36, 38, 40 y 42 . Además observaron que en invierno la grasa contenía más cantidad de ácido mirístico $\left(\mathrm{C}_{14: 0}\right)$ y palmítico $\left(\mathrm{C}_{16: 0}\right)$ y menos de esteárico $\left(C_{18: 0}\right)$ y oleico $\left(C_{18: 1}, n-9\right)$, presentando por lo tanto una temperatura de fusión mayor. Weber et al., (1988a,b) observaron una mayor proporción de TGs de NP menores o iguales a 42 en la leche de invierno respecto a la de verano, ocurriendo lo contrario para los TGs de NP mayores o iguales a 43. Igualmente, Hinrichs et al., (1992) encontraron en la leche de verano fundamentalmente TGs con NP entre 44 y 50 , y entre NP 30 y 42 en la leche de invierno. Los estudios anteriores confirman que la leche de invierno se caracteriza por presentar mayores contenidos de TGs con ácidos grasos saturados, de cadena corta y media, y menores contenidos de TGs con ácidos grasos insaturados y cadena larga, que la leche de verano. Por otra parte se ha observado, en general, que la distribución posicional de los ácidos grasos en los TGs permanece prácticamente constante durante los meses del año (Morrison y Hawke, 1978; Parodi, 1979).

\section{COMPOSICIÓN TRIGLICERÍDICA DEL ACEITE DE PESCADO}

Del total de lípidos, los TGs constituyen la mayor fracción del aceite de pescado, de la cual un 11\% aproximadamente corresponde a fosfolípidos (Bandarra et al., 1997). Entre estos últimos el más abundante es la fosfatidilcolina, que se encuentra entre el 45 y el $60 \%$ del total dependiendo de las especies (Kuksis, A., 1976, Wada, S. et al., 1979). Los aceites de pescado contienen una inmensa variedad de ácidos grasos, sobre todo de cadena media y alta, aunque generalmente se circunscriben a longitudes de cadena entre $\mathrm{C}_{14}$ y $\mathrm{C}_{24}$ átomos de carbono (Laakso et al., 1990). Ya en 1970, Linko y Karinkanta encontraron hasta 14 ácidos grasos con más de 24 átomos de carbono llegando hasta $\mathrm{C}_{32}$, como es el caso del $\mathrm{C}_{32: 6 .}$. Además, esos ácidos grasos pueden contener una variedad enorme también de dobles enlaces, desde 0 hasta 7 . Este amplio rango de ácidos grasos resulta en una mezcla extremadamente compleja de TGs.

La mayor parte de los estudios destinados a caracterizar la composición en TGs del aceite de pescado se han realizado sobre pescados de alto contenido graso, tales como el arenque, la sardina o el hígado de bacalao.

Los aceites de pescado contienen grandes cantidades de ácidos grasos poliinsaturados de cadena larga, que son los que los caracterizan, pero también de saturados. Así, entre el 20 y $45 \%$ de los ácidos grasos corresponde a saturados, mientras que entre 
el 15 y $40 \%$ corresponden a poliinsaturados y alrededor de un $15-20 \%$ a monoinsaturados. Hasta un $3 \%$ del total, corresponde a ácidos grasos de número de carbonos impar, sobre todo a $\mathrm{C}_{15: 0}$ y $\mathrm{C}_{17: 0}$ (Kinsella, 1987, Ackman, 1989, Laakso et al., 1990, McGill y Moffat, 1992, Myher et al., 1996, Pagnucco et al., 1997).

De entre los ácidos grasos saturados que componen el aceite de pescado, destacan por su abundancia el ácido mirístico $\left(\mathrm{C}_{14: 0}\right)$ y el ácido palmítico $\left(\mathrm{C}_{16: 0}\right)$, con contenidos del $10-20 \%$ para el primero y del $15-25 \%$ para el segundo. Otro saturado relevante, además del ácido pentadecanoico $\left(\mathrm{C}_{15: 0}\right)$ y del ácido heptadecanoico $\left(\mathrm{C}_{17: 0}\right)$ es el ácido esteárico $\left(\mathrm{C}_{18: 0}\right)$, aunque siempre por debajo del 5\% (Kinsella, 1987, McGill y Moffat, 1992, Pagnucco et al., 1997). Se ha observado la presencia de los ácidos cáprico y laúri$\operatorname{co}\left(C_{10: 0}\right.$ y $\left.C_{12: 0}\right)$, pero en cantidades inferiores al $1 \%$ (Myher et al., 1996). El ácido oleico $\left(C_{18: 1}, n-9\right)$ representa alrededor del $20 \%$ del total, siendo el más abundante entre los monoinsaturados, aunque se pueden encontrar palmitoleico $\left(C_{16: 1}, n-7\right)$, gadoleico $\left(C_{20: 1}, n-\right.$ 9) y erúcico $\left(\mathrm{C}_{22: 1}, \mathrm{n}-9\right)$ en porcentajes que rondan el $8-15 \%$, e incluso ácido nervónico $\left(C_{24: 1}, n-9\right)$. Los aceites de pescado se conocen por su alto contenido en ácidos grados poliinsaturados de cadena larga de la serie n-3. De entre éstos, se sabe que los ácidos eicosapentaenoico $\left(\mathrm{C}_{20: 5}, \mathrm{n}-3\right)$ y docosahexaenoico $\left(\mathrm{C}_{22: 6}, \mathrm{n}-3\right)$ son los que poseen una mayor actividad favorable para la salud, ya que se relacionan con descensos en la mortalidad debida a enfermedades coronarias (Shekelle, 1985, Kestin, 1990). Cada uno de estos dos ácidos grasos se encuentra en el pescado en proporciones entre el $6 \%$ y el $15 \%$ aunque en general el contenido de ácido eicosapentaenoico $\left(\mathrm{C}_{20: 5}, \mathrm{n}-3\right)$ es superior. El resto del elevado número de ácidos grasos poliinsaturados se encuentra generalmente por debajo del $5 \%$ (Laakso et al., 1990, McGill y Moffat, 1992, Pagnucco et al., 1997).

Dada su complejidad y por consiguiente las dificultades de su análisis, existen muy pocos trabajos referentes a la composición en especies moleculares de TGs del aceite de pescado. Así pues, a diferencia de los aceites vegetales, el estudio de las especies moleculares de TGs del aceite de pescado se encuentra en sus inicios, y por tanto aún queda mucho por hacer. El grupo de Christie ha desarrollado un intenso trabajo en el campo de la determinación de TGs en aceites de pescado mediante HPLC con columnas impregnadas con iones de plata $\mathrm{CAg}^{+}$HPLC) (Christie, 1988, Laakso et al., 1990, Laakso y Christie, 1991).

La cromatografía $\mathrm{Ag}^{+}-\mathrm{HPLC}$ separa las moléculas en base a su grado de insaturación, la distribución de los dobles enlaces dentro de la molécula, la configuración y distribución de los dobles enlaces dentro de cada uno de los ácidos grasos y la posición este- reoespecífica en que los ácidos grasos se encuentran unidos al glicerol. El mecanismo de separación se basa en el establecimiento de enlaces débiles entre los iones de plata y los electrones $\pi$ de los dobles enlaces de las moléculas (Nikolova-Bamyanova, 1992). Así, Laakso et al., (1990) separaron hasta 26 fracciones del aceite de arenque y McGill y Moffat (1992), recogieron 18 de hígado de bacalao y 19 de aceite de sardina. Las fracciones que se obtienen de este modo contienen TGs con diferentes grados de insaturación. La primera es aquélla compuesta por moléculas trisaturadas (SSS), a continuación el orden de elución es SSM, SMM, SSD, MMM, SMD, MMD, etc., donde $S, M$ y $D$ se refieren a ácidos grasos saturados, monoinsaturados y diinsaturados, respectivamente. $A$ medida que el número de insaturaciones aumenta, las combinaciones se complican y aparecen TGs que contienen ácidos grasos con una variedad muy grande en cuanto a su número de dobles enlaces, dificultando el análisis, se trata de ácidos grasos triinsaturados, tetrainsaturados, pentainsaturados y hexainsaturados, $\mathrm{Tr}, \mathrm{Te}, \mathrm{P}$ y $\mathrm{H}$, respectivamente. El aceite de arenque atlántico, según Laakso et al., (1990), contiene como fracción mayoritaria la de MMS, con un $14,5 \%$. Otras fracciones representan alrededor de un $10 \%$, pero su composición en ácidos grasos es sumamente compleja, aunque entre otros, contenían porcentajes cercanos al $30 \%$ de $\mathrm{C}_{20: 5}, \mathrm{n}-3$ o $\mathrm{C}_{22: 6}, \mathrm{n}-3$. Un $8 \%$ corresponde a la fracción SSM, cuyos ácidos grasos principales son palmítico $\left(C_{16: 0}\right)$, mirístico $\left(C_{14: 0}\right)$, oleico $\left(C_{18: 1}, n-9\right)$ y erúcico $\left(\mathrm{C}_{22: 1}, \mathrm{n}-9\right)$. La fracción SSS, con un $2 \%$, contiene palmítico $\left(\mathrm{C}_{16: 0}\right)$ y mirístico $\left(\mathrm{C}_{14: 0}\right)$ como ácidos grasos principales.

Otros autores han probado la realización del análisis del aceite de pescado mediante RP-HPLC. Pagnucco et al., (1997) alcanzaron la separación de 19 fracciones de aceites de sardina, anchoa y arenque pero no de especies moleculares, con NP que se encontraban entre 36 y 50 . Bergqvist y Kaufmann (1993) consiguieron resolver 35 picos individuales de un aceite de pescado, que se encontraban entre NEC 35.24 y 48.49 , calculado por estos autores a partir de un polinomio de quinto grado, que relacionaba el NEC con el valor del índice de polaridad de Snyder ( $\left.P^{\prime}\right)$, que da una idea del ambiente de polaridad que rodea a cada molécula de TG en un momento dado de la elución cromatográfica. Desafortunadamente solamente fueron capaces de identificar 3 de ellos: dipalmitoil-linoleoil-glicerol (PPL), dipalmitoil-oleoil-glicerol (PPO) y tripalmitina (PPP). Finalmente, Perona y Ruiz-Gutiérrez (1998d) han estudiado recientemente la composición molecular de los TGs de la grasa de sardina, siendo capaces de separar 65 picos cromatográficos mediante RP-HPLC, con NEC entre 29.4 y 52.0, de los cuales 59 fueron identificados tras separar la grasa en 4 fracciones mediante cromatografía en capa 
fina impregnada con iones de plata $\left(\mathrm{Ag}^{+}-\mathrm{TLC}\right)$ y analizar cada una de las fracciones por RP-HPLC. En la mayoría de los casos cada uno de los picos contenía solamente un TG y en ningún caso contenía más de dos. El TG más abundante en el aceite de sardina resultó ser la trimiristina (MMM) con un $8.22 \%$ del total. Se encontraron así mismo cantidades apreciables de especies de dioleoil-acil-glicerol (OOP, OOE), dipalmitoil-acil-glicerol (PPO,
PPPo), dipalmitoleoil-oleoil-glicerol (PoPoO) and dieicosapentaenoil-palmitoil-glicerol (EEP), siempre por debajo del $6 \%$. La peor resolución de TGs fue la de aquellos que contenían ácidos eicosapentaenoico $\left(\mathrm{C}_{20: 5}, \mathrm{n}-3\right)$ o docosahexaenoico $\left(\mathrm{C}_{22: 6}, \mathrm{n}\right.$ 3) (DDpiM/EDpM, MMD/MME y PoOD/PoOE). Las mismas condiciones se emplearon posteriormente con un aceite de pescado, dando como resultado el cromatograma de la Figura 2.

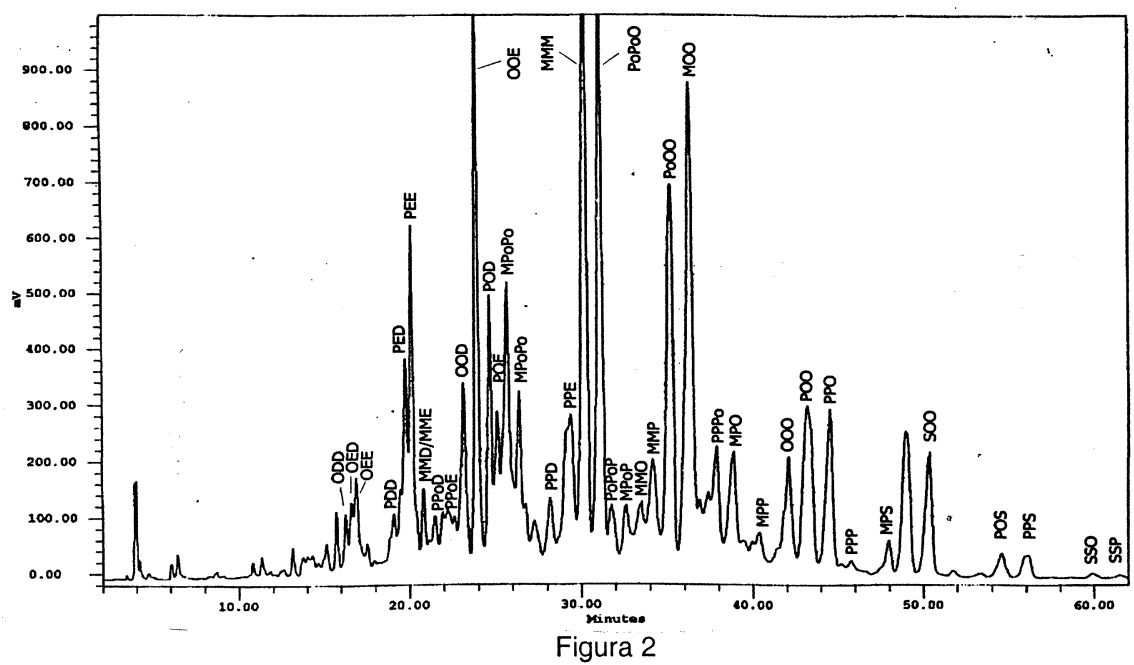

Cromatograma de triglicéridos de aceite de pescado mediante HPLC. Separación llevada a cabo a $40{ }^{\circ} \mathrm{C}$,

empleando una columna Spherisorb ODS-2 ( $3 \mu \mathrm{m}, 250 \times 4.6 \mathrm{~mm})$. Gradiente de acetona en acetonitrilo $20-45 \%$ de acetona en $12 \mathrm{~min}$ y hasta $80 \%$ en $58 \mathrm{~min}$. Disolvente de inyección n-hexano, $10 \mu \mathrm{l}$.

$\mathrm{M}=$ ácido mirístico. 14:0; $\mathrm{P}=$ ácido palmítico. 16:0; $\mathrm{S}=$ ácido esteárico. 18:0; $\mathrm{Po}=$ ácido palmitoleico. 16:1, n-7; $\mathrm{O}=$ ácido oleico: 18:1, $\mathrm{n}$ 9; $\mathrm{E}=$ ácido eicosapentaenoico. 20:5, n-3. D = ácido docosahexaenoico. 22:6, n-3 Triglicéridos: $P P P=$ tripalmitoil-glicerol; $P P D=$ dipalmitoil-docosahexenoil-glicerol; POS = palmitoil-oleoil-estearoil-glicerol, etc.

La combinación de $\mathrm{Ag}^{+}$-HPLC y RP-HPLC para el estudio de la composición triglicerídica del aceite de arenque (uno de los más complejos) por el grupo de Christie (Laakso, et al., 1991), obtuvo un resultado bastante satisfactorio, si bien no fue posible determinar especies moleculares individuales. Se separaron 11 fracciones mediante $\mathrm{Ag}^{+}-\mathrm{HPLC}$, que posteriormente fueron inyectadas individualmente en un sistema de HPLC con columna de fase reversa. Como resultado obtuvieron un total de 130 fracciones con NEC comprendidos entre 30.0 y 53.6, de las que se determinó su composición en ácidos grasos.

En general, los aceites naturales contienen solamente un ácido graso poliinsaturado de cadena larga por molécula (Myher et al., 1996). Los aceites vegetales, presentan ácidos grasos poliinsaturados de cadena $\mathrm{C}_{18}$ en la posición sn-2, con los poliinsaturados de cadena $\mathrm{C}_{20}$ y $\mathrm{C}_{22}$ en las posiciones exteriores. Por su parte, los aceites de pescado contienen ácidos grasos poliinsaturados de cadena larga en la posición central, mientras que los ácidos monoenoicos ocupan las posiciones sn-1 y sn-3 (Ruiz-Gutiérrez y Barrón, 1995b). En el aceite de hígado de bacalao, el ácido docosahexaenoico $\left(\mathrm{C}_{22: 6}, \mathrm{n}-3\right)$ se localiza efectivamente en la posición sn-2, si bien el eicosapentaenoico ocupa tanto la sn-2 como la sn-3. El oleico y el gadoleico $\left(C_{18: 1}, n-9\right.$ y $\left.C_{20: 1}, n-9\right)$ se encuentran en las posiciones sn- 1 y sn- 3 , mientras que los ácidos grasos saturados ocupan las posiciones sn-1 y sn-2 (Brockerhoff et al., 1968, Ruiz-Gutiérrez y Barrón, 1995b). El mismo principio tiene lugar en el aceite de arenque y de caballa, aunque la posición del ácido oleico puede ser también en gran medida la central, la misma que tienden a ocupar los ácidos grasos saturados (Brockerhoff et al., 1968). En el aceite de atún, los saturados ocupan las posiciones sn-1 y sn-2, los ácidos monoenoicos las exteriores y los poliinsaturados de cadena larga la sn-2, pero también la sn-3 (Myher et al., 1996).

La composición del aceite de pescado varía apreciablemente dependiendo de la especie que se considere, pero también en función de la época del año, puesto que se ve afectada por alteraciones en la alimentación, en la temperatura del agua y por el ciclo biológico en que se encuentre el animal. De entre los componentes de la grasa, son los TGs los que más se ven afectados, por los cambios en la dieta. Bandarra et al., (1997) desarrollaron un estudio acerca de las variaciones estacionales en la composición lipídica del aceite de sardina. Encontraron que los niveles más 
altos de ácidos grasos poliinsaturados, incluyendo eicosapentaenoico $\left(\mathrm{C}_{20: 5}, \mathrm{n}-3\right)$ y docosahexaenoico $\left(C_{22: 6}, n-3\right)$, tenían lugar al finalizar el verano, cuando el pescado tenía mayor cantidad de grasa. Sin embargo el porcentaje relativo de estos ácidos grasos respecto al resto era más alto al principio de la primavera, una vez consumida la grasa durante el invierno. No se observaron modificaciones apreciables en el contenido de los ácidos grasos saturados ni de los monoinsaturados.

\section{COMPOSICIÓN TRIGLICERÍDICA DE LA GRASA HEPÁTICA}

Existe una amplia evidencia de que la denominada dieta mediterránea está asociada a una baja incidencia de enfermedad coronaria. Este efecto ha sido atribuido a un alto contenido de ácidos grasos monoinsaturados en la dieta, procedentes en su mayoría del aceite de oliva (Fidanza et al., 1970; Colquhoun, 1992; Heyden, 1994). De hecho, el ácido graso más abundante en el aceite de oliva, ácido oleico $(18: 1, n-9)$, se ha relacionado con reducciones en el colesterol plasmático total y de lipoproteínas de baja densidad (LDL) sin una reducción proporcional de los niveles de colesterol de lipoproteínas de alta densidad (HDL) (Grundy y Denke, 1990). Recientemente está cobrando importancia la estructura de las moléculas de TGs que componen la dieta y sus efectos fisiológicos (Yand et al., 1995, Lehner y Kuksis, 1996, Ruiz-Gutiérrez et al., 1998, Perona y Ruiz-Gutiérrez, 1998c). El hígado es el órgano en el que los efectos de la estructura y composición de los TGs de la dieta son más relevantes, puesto que es, junto con el intestino, el más activo en el metabolismo de TGs. En este órgano, se produce la resíntesis de los TGs, a partir de ácidos grasos procedentes de la dieta y de las reservas corporales. Los TGs son posteriormente transportados a los tejidos periféricos vía lipoproteínas de muy baja densidad (VLDL). A fin de incorporar los ácidos grasos al metabolismo lipídico celular, los TGs son hidrolizados mediante la lipoproteína lipasa, enzima que ha demostrado especificidad por los ácidos grasos localizados en las posiciones 1 y 3 de la molécula de glicerol (Bracco, 1994). Estudios recientes han señalado que la ingesta de ácidos grasos mono y poliinsaturados afecta a la composición de las VLDLs y a la expresión de enzimas relacionadas con su catabolismo (McNamara, 1995). La estructura de los TGs de las VLDLs depende pues de la composición y el comportamiento de las especies moleculares de TGs en el hígado.

La principal característica de la grasa hepática es la elevada cantidad de fosfolípidos que contiene $(60 \%)$, lo que dificulta enormemente la determinación de los TGs, ya que se impone la inclusión en el proceso de una etapa para su aislamiento. Además contiene una cantidad de colesterol que ronda el $8 \%$, lo que deja el contenido de TGs alrededor del $30 \%$ aproximadamente, aunque depende en gran medida de la dieta (Muriana et al., 1992, Perona y Ruiz-Gutiérrez, 1998c).

La composición en ácidos grasos del hígado, y por tanto la composición en TGs, es en buena parte reflejo de la dieta. Por tanto, no se puede establecer una composición única de ácidos grasos sin tener en cuenta qué dieta se ha consumido. Una dieta rica en aceite de oliva por ejemplo, proporciona una gran cantidad de ácido oleico $\left(\mathrm{C}_{18: 1}, \mathrm{n}-9\right)$, que se acumula en el hígado, dando como resultado hasta casi un $30 \%$ de este ácido graso respecto al total (Perona et al., 1998a,b,c).

Los trabajos desarrollados hasta la fecha han registrado una amplia variedad de especies moleculares de TGs en el hígado. Perona et al., (1998b) identificaron 31 especies mediante RP-HPLC con detector lightscattering. Los ácidos oleico $\left(C_{18: 1}, n-9\right)$, linoleico $\left(\mathrm{C}_{18: 2}, \mathrm{n}-6\right)$ y palmítico $\left(\mathrm{C}_{16: 0}\right)$ componían los TGs mayoritarios: palmitoil-oleoil-linoleoil-glicerol (POL), dioleoil-linoleoil-glicerol (OOL), palmitoil-dioleoil-glicerol (POO), trioleína (OOO), palmitoil-dilinoleoil-glicerol (PLL), dipalmitoil-oleoil-glicerol (PPO) y oleoil-dilinoleoil-glicerol (OLL), mientras que entre los minoritarios se identificaron TGs que contenían ácido araquidónico $\left(\mathrm{C}_{20: 4}, \mathrm{n}-6\right)$ y docosahexaenoico $\left(\mathrm{C}_{22: 6}, \mathrm{n}-3\right)$. Otros autores también han estudiado en el hígado la composición de TGs mediante RP-HPLC y light-scattering. Huang et al., (1992) separaron 19 especies moleculares de TGs utilizando para ello dos columnas de fase reversa dispuestas en serie y un detector lightscattering. En este caso las especies más importantes eran aquellas que contenían ácido linoleico $\left(\mathrm{C}_{18: 2}, \mathrm{n}-6\right)$ debido a que la dieta contenía un $80 \%$ de este ácido graso. Chen y Cunnane (1993) han estudiado la composición triglicerídica del hígado, separando hasta 17 picos cromatográficos en la grasa hepática de ratas alimentadas con dietas enriquecidas en ácido linoleico $\left(\mathrm{C}_{18: 2}, \mathrm{n}-6\right)$ si bien no se identificaron especies moleculares. Por esta razón, en este caso también las especies más abundantes eran las que contenían este ácido graso. Yang et al., (1995) compararon las especies moleculares de TGs del hígado de rata con la de las VLDL observando grandes similitudes tanto en la composición en ácidos grasos como en especies de TGs. En este trabajo se encontraron también TGs que contenían ácidos grasos poliinsaturados de cadena larga $\left(\mathrm{C}_{20: 4}, \mathrm{n}-6\right.$ y $\left.\mathrm{C}_{22: 6}, \mathrm{n}-3\right)$, aunque los mayoritarios fueron el $\mathrm{PLL}$, POL/SLL y el POO/SOL, que entre los 3 sumaban el $51 \%$. La Figura 3 muestra el cromatograma resultante del análisis de triglicéridos del hígado de rata determinados empleando una columna de fase reversa y detector light-scattering. 


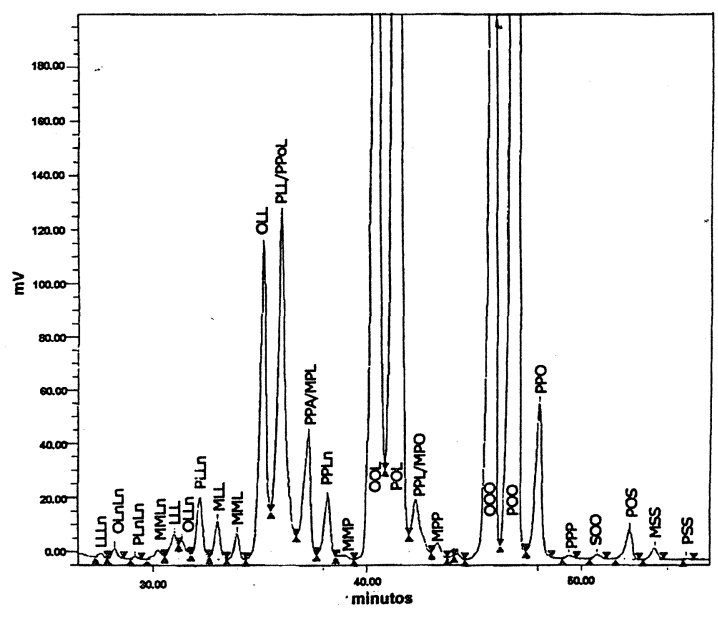

Figura 3

Cromatograma de triglicéridos de la grasa hepática mediante HPLC. Separación llevada a cabo a $40^{\circ} \mathrm{C}$, empleando una columna Spherisorb ODS-2 $(3 \mu \mathrm{m}, 250 \times 4.6 \mathrm{~mm})$. Gradiente de acetona en acetonitrilo $20-45 \%$ de acetona en 4 min y hasta $80 \%$ en 36 min. Disolvente de inyección $\mathrm{n}$-hexano, $10 \mu \mathrm{l}$.

$M=$ ácido mirístico. 14:0; $P=$ ácido palmítico. 16:0; $\mathrm{S}=$ ácido esteárico. 18:0; $\mathrm{Po}=$ ácido palmitoleico. 16:1, $\mathrm{n}-7 ; \mathrm{O}=$ ácido oleico. 18:1, n-9; $\mathrm{L}=$ ácido linoleico. 18:2, n-6; $\mathrm{Ln}=$ ácido linolénico. 18:3, n-3. Trigicéridos: $\mathrm{PPP}=$ tripalmitoilglicerol, $\mathrm{PPL}=$ dipalmitoil-linoleoil-glicerol; POS = palmitoil-oleoil-estearoil-glicerol, etc.

Existen pocos estudios relativos al estudio de las posiciones estereoespecíficas en que los ácidos grasos que componen los TGs del hígado esterifican la molécula de glicerol. Innis et al., (1996) comprobaron en el hígado de cerdo, que al contrario de lo que sucede en el tejido adiposo de este animal, el ácido palmítico $\left(\mathrm{C}_{16: 0}\right)$ no ocupa la posición sn-2. Efectivamente, esta posición central es ocupada por los ácidos oleico $\left(C_{18: 1}, n-9\right)$ y linoleico $\left(C_{18: 2}, n-6\right)$. Este hecho ha sido observado también en el hígado de rata por Yang et al., (1995 y 1996). Según estos autores, el ácido palmítico $\left(C_{16: 0}\right)$, así como otros saturados, tienden a situarse en la posición sn-1, si bien el esteárico $\left(\mathrm{C}_{18: 0}\right)$ también podría ocupar la posición sn-3. Los ácidos oleico y linoleico $\left(C_{18: 1}, n-9\right)$ y $\left(C_{18: 2}, n-6\right)$ se localizan preferentemente en la posición central, mientras que los ácidos poliinsaturados de cadena larga se encuentran casi exclusivamente en la posición sn-3.

\section{CONCLUSIÓN}

Actualmente la técnica de HPLC en fase reversa, es la que mejores resultados ofrece para el análisis de las especies moleculares de TGs en grasas tan complejas como las descritas en el presente artículo. Mediante esta técnica, utilizando fases móviles, en general compuestas por gradientes de acetona en acetonitrilo, y con detección light-scattering, es posible separar en moléculas individuales los TGs de la grasa láctea, el aceite de pescado y la grasa hepática, reduciendo al mínimo, e incluso eliminando, el número de pares críticos sin resolver. Sin embargo, en la mayoría de los casos es necesaria la participación de otras técnicas, como la cromatografía de gases o la espectrometría de masas para la identificación de los picos cromatográficos. Por otra parte, aún queda largo plazo para el establecimiento de un método simple y rápido que permita separar isómeros posicionales de TGs.

\section{BIBLIOGRAFÍA}

Ackman, R.G. (Ed.). (1989).—«Marine Biogenic Lipids».Fats and Oils. I, 139. Boca Raton Florida., CRC Press Inc.

Alais, Ch. (1985)._- «Ciencia de la leche. Principios de la técnica lechera".-Barcelona., Ed. Reverté, S.A.

Anifantakis, E.M. (1986)._- "Comparison of the physicochemical properties of ewes and cows milk.».--Int. Dairy Fed. Bull., 202: 42-53

Arumughan, C., Narayanan, K. M. (1982).-Triacylglycerol composition of cow milk fat».-J. Food Sci. Technol., 19: $71-74$

Baer, R.J. (1991).— - Alteration of the fatty acid content of milk fat".-J. Food Protec., 54: 383-836.

Bandarra, N.M., Batista, I. Nunes, M. L., Empis, J. M., Christie, W. W. (1997). - Seasonal changes in lipid composition of sardine (Sardina pilchardus)".- J. Food Sci., 62: 40-42.

Banks, W., Christie, W. W. Clapperton, J. L. Girdler, A. K. (1987).- - The trisaturated glycerides of bovine milk fat».-J. Sci. Food Agr., 39: 303-316.

Barbano, B.M., Sherbon, J. W. (1979)._«Stereospecific analysis of high melting triglycerides of bovine milk fat and their biosynthetic origin».-J. Dairy Sci., 58: 1-8.

Barrón, L.J.R., Hierro, M.T.G. Santa-María, G. (1990)."HPLC and GLC analysis of the triglyceride composition of bovine, ovine and caprine milk fat".-J. Dairy Res., 57: 517-526.

Barrón, L.J.R., Santa-María, G. (1989).— «HPLC analysis of complex mixtures of triglycerides using gradient elutions and an ultraviolet detector".-Chromatogr., 28: $183-188$.

Beaulieu, A.D., Palmquist, D.L. (1995).—-Differential effects on high fat diets on fatty acid composition in milk on Jersey and Holstein cows».-J. Dairy Sci., 78: 1336-1344.

Bencini, R., Pulina, G. (1997).- «The quality of sheep milk: a review».-Aust. J Exp. Agr., 37: 485-504.

Bergqvist, B.H.J., Kaufmann P. (1993).— «A multivariate optimization of triacylglycerol analysis by highperformance liquid chromatography".-Lipids, 28: 667-675.

Blank, M.L., Privett, O. S. (1964).- «Structure of milk fat triglycerides».-J. Dairy Sci., 47: 481-189.

Bracco, U. (1994).- - «Effects of triglyceride structure on fat absorption».-Am. J. Clin. Nutr., 60 (suppl): 1002S1009S.

Brockerhoff, H., Hoyle, R. J. Hwang, P. C., Litchfield C. (1968). - «Positional distribution of fatty acids in depot triglycerides of aquatic animals".--Lipids, 3: 24-29. 
Caboni, M.F., Massari, A., Lercker, G., Losi G. (1982)."Composizione del grasso nel latte: nota I. I lipidi apolari».-Sci. Tecn. Lattiero-Casearia, 33: 426-442.

Carelli, A.A., Cert, A. (1993)._- "Comparative study of the determination of triacylglycerol in vegetable oils using chromatographic techniques".-J. Chromatogr., 63: 213-222.

Charlesworth, J.M. (1978).—-Evaporative analyzer as a mass detector for liquid chromatography".-Anal. Chem., 50: 1414-1420.

Chazal, M.P., Chilliard, Y. (1986).—«Effect on stage of lactation, stage of pregnancy, milk yield and herd management on seasonal variation in spontaneous lipolysis in bovine milk».-J. Dairy Res., 53: 529-538.

Chen, Z. Y., Cunnane, S. C. (1993).-Fasting-induced remodelling of hepatic triacylglycerols".-J. Nutr. Biochem., 4: 421-425.

Christie, W.W. (1980).- «The effects of diet and other factors on the lipids composition of ruminant tissues and milk».-Prog. Lipid Res., 17: 245-277.

Christie, W.W. (1987).- «High-performance liquid Chromatography of Lipids: A practical Guide. 183-208».--Oxford, Pergamon Press.

Christie, W.W. (1988). - «Separation of molecular species of triacylglycerols by high-performance liquid chromatography with a silver ion column".-J. Chromatogr, 454: 273-284.

Christie, W.W., Clapperton J. L. (1982).- «Structures of the triglycerides of cows' milk, fortified milks (including infant formulae), and human milk».-J. Soc. Dairy Technol., 35: 22-24.

Colquhoun, D. (1992). - «Monounsaturates and heart disease». In:-Essential fatty acids and eicosanoids. Edited by Sinclair A, Gibson R. Champaign: American Oil Chemist's Society; pp. 279-286.

Contarini, G., Toppino, P. M. (1995)._- Lipolysis in Gorgonzola cheese during ripening".-Int. Dairy Journal, 5: 141-155.

Cullinane, N., Aherne, S., Connolly, J. F., Phelan J. A. (1984). - "Seasonal variation in the triglyceride and fatty acid composition of irish butter».-J. Food Sci. Technol., 8: 1-12.

Faulkner, A., Pollock H. T. (1989).-—Changes in concentration of metabolites in milk from cows fed on diets supplemented with soyabean oil or fatty acids".-J. Dairy Res., 56: 170-183.

Fidanza, F., Puddu, V., Imbombo, A.B., Menotti, A., Keys, A. (1970).- "Coronary heart disease in seven countries VII" .-Circulation, 41 (suppl): 163-175.

Frede, E., Thiele, H. (1987).— «Analysis of milk fat by HPLC".-J. Am. Oil Chem. Soc., 64: 521-528.

Galvano, G., Scerra, V. (1984).—«Research on the milk of domestic ruminants: 1 . Considerations and comparisons on fatty acids of the lipidic fraction of the milk of sheep, goats, cows, and buffaloes".-World Review of Animal Production, XX: 71-80.

Gibson, J.P. (1981).- «The potential for genetic change in mlik fat composition".-J. Dairy Sci., 74: 3258-3266.

Goiffon, J.P., Reminiac, C., Furon, D. (1981)."Application de la chromatographie liquide haute performance a l'analyse des triglycérides des corps gras. II- Grandeurs de rétention des triglycérides"Rev. Franc. Corps Gras, 28: 199-206.

González-Llano, D., Ramos, M. (1989).- “Composición química de la leche de oveja».-Ovis, 2: 9-19.

Gresti, J., Bugaut, M., Maniongui, C., Bezard, J. (1993)."Composition of molecular species of triacylglycerols in bovine milk fat».-J. Dairy Sci., 76: 1850-1869.
Grundy, S.M., Denke, M.A. (1990).—-Dietary infiuences on serum lipids and lipoproteins».-J. Lipid Res.; 31: 1149-1172.

Guiochon, G., Moysan, A., Holley, C. (1988).- J. Liq. Chromatogr. And Rel. Technol., 11, 2547.

Hawke, J.C., Taylor, M.W. (1983).- «Influence of nutritional factors on the yield composition and physical properties of milk fat».-Dev. Dairy Chem., 2: 37-81.

Heyden, S. (1994).- « Polyunsaturated and monounsaturated fatty acids in the diet to prevent coronary heart disease via cholestrol reduction».-Ann Nutr Metab., 38: 117-122.

Herslöf, B., Podlaha, G., Toregard, B. (1979).—-HPLC of triglycerides».-J. Am. Oil Chem. Soc., 56: 864-866.

Hierro, M.T.G., Tomás, M.C., Fernández-Martín, F., SantaMaría, G. (1992).—-Determination of the triglyceride composition of avocado oil by high- performance liquid chromatography using a light- scattering detector».-J. Chromatogr., 607: 329-338.

Hinrichs, J., Heinemann, U., Kessler, H.G. (1992)."Differences in the composition of triglycerides in summer and winter milk fat".-Milchwiss., 47: 495-498.

Huang, Y. S., Lin, X., Smith, R.S., Redden, P.R., Ells, G.W., Horrobin, D.F. (1992). - «Effect of fasting on the distribution of triacylglycerol molecular spieces in rat liver».-Med. Sci. Res., 20: 691-692.

Itabashi, Y., Takagi, T. (1986).— «High-performance liquid chromatography separation of monoglycerol enantiomers on a chiral stationary phase».-Lipids, 21: 413-416.

Itabashi, Y., Myher, J.J., Kuksis, A. (1993).—«Determination of positional distribution of short-chain fatty acids in bovine milk fat on chiral columns".-J. Am. Oil Chem. Soc., 70: 1177-1181.

Innis, S.M., Dyer, R., Quinlan, P.T., Diersen-Schade, D. (1996).- - «ietary triacylglycerol structure and saturated fat alter plasma and tissue fatty acids in piglets».-Lipids, 31: 497-505.

Jensen, R.G., Clark, R.W. (1988).—-Lipid composition and properties. N.P. Wong (ed)". - Fundamentals of dairy chemistry, 3rd ed. 171-213. New York, Van Nostrand Reinhold Co.

Jensen, R.G., Ferris, A. M., Lammi-Keeje, C. J. (1991).— «Symposium: Milk fat composition, function, and potential for change. The composition of milk fat».-J. Dairy Sci., 74: 3228-3243.

Kaluzny, M.A., Duncan L.A., Merrit M.V. y Epps D.E. (1985).— - «apid separation of lipid classes in high yield and purity using bonded phase columns".-J. Lipid Res, 26: 135-40.

Keys, A., Menotti, A., Karvonen, M.J., Aravanis C., Blackburn, H., Buzina, R., Cjordjevic, B.A., Dontas, S., Fidanza, F., Keys, M.H., Kromhout, D., Nedeljkovic, S., Punsar, S., Seccareccia, F., Toshima, H. (1986)."The diet and 15-year death rate in the seven countries study».-Am. J. Epidemiol, 124: 903-915.

Kinsella, J.E. (Ed.) (1987)._- "Seafoods and Fish Oils in Human Health and Disease".-Edible Fish Oils Processing and Technology. New York and Basel., Marcel Dekker. Inc.

Klobasa, F., Farries, E. (1986).- - "Changes of the fatty acid spectrum in milk fat of cows during lactation".-Milchwiss, 41: 146-148.

Kuksis, A., Marinetti, G.E. (1976).——Lipid chromatographic Analysis".-215. New York, Marcel Dekker.

Kuksis, A., Marai, L., Myher, J.J., Cerbulis, J., Farrell, H.M. (1986). — «Comparative study of the molecular species of chloro-propanediol diesters and triacylglycerols in milk fat».-Lipids, 21: 183-190. 
Laakso, P., Christie, W.W., Pettersen, J. (1990)."Analysis of North Atlantic and Baltic fish oil triacylglycerols bu high-performance liquid chromatography with a silver ion column».-Lipids, 25: 284-291.

Laakso, P., Christie, W.W. (1991).—«Combination of silver ion and reversed-phase high.performance liquid chromatography in the fractionation of herring oil triacylglycerols".-J. Am. Oil Chem.Soc., 68: 213.

Lehner, R., Kuksis, A. (1996). - «Biosynthesis of triacylglycerols».Prog. Lipid Res., 35: 169-201.

Linko, R.R., Karinkanta, H. (1970)._- «Fatty Acids of Long Chain Length in Baltic Herring Lipids".- J. Am. Oil Chem. Soc., 47: 42-46.

Lomascolo, A., Dubreucq, E., Perrier, V., Galzy, P. (1994). - «Observations concerning the composition of fatty acids in dairy products".-Milchwiss, 49: 559-561.

Lozano, Y. (1983).- «Analyse des triglicérides de l'huile d'avocat par CLHP en phase inverse".-Rev. Franc. Corps Gras., 30: 333.

Lund, P. (1988).— - Analysis of butterfat triglycerides by capillary gas chromatography".-Milchwiss, 43: 159-161.

Marjanovic, N., Jankovits, I., Turkulov, J., Karlovic, D., Caric, M., Milanovic, S., Zagorac, M. (1984).«Determination of the triglyceride composition of milk fat by gas chromatography".-Mljekarstvo, 34: 67-75.

McGill, A.S., Moffat, C.F. (1992). - «A study of the composition of fish liver and body oil triglycerides". Lipids, 27: 360-370.

McNamara, D.J. (1995)._ «Dietary fatty acids, lipoproteins and cardiovascular disease».-Adv. Food. Nutr. Res., 36: 253-352.

Molkentin, J., Precht, D. (1994).—«Comparison of packed and capillary columns for quantitative gas chromatography of triglycerides of milk fat".Chromatogr., 39: 265-270.

Muriana, F.J.G., Vázquez, C.M. y Ruiz-Gutiérrez V. (1992).«Fatty acid composition and properties of the liver microsomal membrane of rats fed diets enriched with cholesterol".-J. Biochem., 112: 562-567.

Myher, J.J., Kuksis, A., Geher, K., Park, P.W., DiersenSchade, D.A. (1996)._- «Stereospecific analysis of triacylglycerols rich in long-chain polyunsaturated fatty acids".-Lipids, 31: 207-215.

Nájera, A.I., Barcina, Y., De Renobales, M., Barron, L.J.R. (1998).- «Determination of the triacylglycerol compositon of Idiazabal cheese".-Chromatogr., 47: 576-579.

Nikolova-Damyanova, B. (1992).- «Silver-lon Chromatography and Lipids".-Christie, W. W. ed. Advances in Lipid Methodology-One. 181-237. Dundee, The Oily Press.

Nurmela, K.V.V., Satama, L.T. (1988)._- «Quantitative analysis of triglycerides by high-performance liquid chromatography using non-linear gradient elution and flame ionization detection».-J. Chromatogr., 435, 139.

Pacheco, Y.M., Pérez-Camino, M.C., Cert, A., Montero, E., Ruiz-Gutiérrez, V. (1998).- - «Determination of the molecular species composition of diacylglycerols in human adipose tissue by solid-phase extraction and gas chromatography on a polar phase».-J. Chromatogr. B (en prensa).

Pagnucco, C., Toschi, T.G., Serrazanetti, G.P. (1997).—«A chromatographic study on triacylglycerol composition of fish oils".-Rev. Ital. Sost. Grasse, 74: 7-12.

Palmquist, D.L., Beaulieu, A.D., Barbano, D.M. (1993).«ADSA foundation symposium: milk fat synthesis and modification. Feed and animal factors influencing milk fat composition».-J. Dairy Sci., 76: 1753-1771.
Parodi, P.W. (1970)._- «Fatty acid composition of Australian butter and milk fats".-Aust. J. Diary Technol., 25: 200-205.

Parodi, P.W. (1980). - «Separation of milk fat triglycerides into classes by silver ion adsorption thin-layer chromatography".-Aust. J. Diary Technol., 35: 17-22.

Parodi, P.W. (1982)._-«Positional distribution of fatty acids in the triglyceride classes of milk fat».-J. Dairy Res. 49: 73-80.

Parodi, P.W. (1983).— «Relationship between fatty acid composition and triglyceride structure of bovine milk fat".-J. Dairy Res., 50: 443-447.

Pauls, R.E. (1983).— - A time normalization study of the separation of olive oil triglycerides".-J. Am. Oil Chem. Soc., 60: 819-822.

Pei, T., Henly, R., Ramachandran, S. (1975).- «New application of high pressure reversed-phase liquid chromatography in lipids". - Lipids, 10: 152-156.

Pellegrini, O., Remeuf, F., Rivemale, M., Barillet, F. (1997)._- «enneting properties of milk from individual ewes: influence of genetic and non-genetic variables, and relationship with physicochemical characteristics».J. Dairy Res., 64: 355-366.

Perona, J.S., Barrón, L.J.R., Ruiz-Gutiérrez, V. (1998a)."Determination of Rat Liver Triglycerides by Gas-Liquid Chromatography and Revesed-Phase High-Performance Liquid Chromatography".-J. Chromatogr., 706: 176-179.

Perona, J.S., Barrón, L.J.R., Ruiz-Gutiérrez , V. (1998b).«Molecular prediction of Rat Liver Triglycerides by High-Performance Liquid Chromatography».-J. Liq. Chromatogr. and Rel. Technol., 21: 1185-1197.

Perona, J.S., Ruiz-Gutiérrez, V. (1998c).—«Two highly monounsaturated oils, olive oil and high-oleic sunflower oil, induce different triacylglycerol molecular species distribution in rat liver».-Nutr. Res., (en prensa).

Perona, J.S., Ruiz-Gutiérrez, V. (1998d).—«Triacylglycerol molecular species composition of sardine oil by reversed-phase high-perfomance liquid chromatography". J. Liq. Chromatogr. Relat. Technol., (en prensa).

Plattner, R.D., Spencer, G.F., Kleinman, R. (1977).«Triglyceride separation by reversed-phase highperformance liquid chromatography».-J. Am. Oil Chem. Soc., 54: 511-515.

Precht, D., Frede, E.: (1994).— — Determination of the solid fat content in milk fat by gas chromatographic triglyceride analysis".-Fat Sci. Technol., 9: 324-330.

Ramos, M., Juárez, M. (1986).— «Métodos analíticos para la identificación de mezclas de leche de diferentes especies".-R.E.L., 7: 19-31.

Riguezza, M., Guiochon, G. (1967).-—J. Liq. Chromatogr. Rel. Technol».-11, 1967. 1988.

Ruiz-Gutiérrez, V., Prada, J.L., Pérez-Jiménez, F. (1993)."Determination of fatty acid and triacylglycerol composition of human very-low-density lipoproteins".J. Chromatogr. B, 622: 117-124.

Ruiz-Gutiérrez, V., Barrón, L.J.R. (1995a).— «Methods for the analysis of triacylglycerols».-J. Chromatogr. B, 671: 133-168.

Ruiz-Gutiérrez, V., Barrón, L.J.R. (1995b).—«Análisis estereospecífico de los triacil-sn-gliceroles de las grasas naturales por métodos enziáticos y cromatografia líquida de alta eficacia».-Grasas y Aceites, 46: 376-382.

Ruiz-Gutiérrez, V., Morgado, N., Prada, J.L., PérezJiménez, F., Muriana, F. J.G. (1998).—-Composition of human VLDL triacylglycerols after ingestion of olive 
oil and high-oleic sunflower oil.».-J. Nutr., 128: 570-576.

Ruiz-Sala, P., Hierro, M.T.G., Martínez-Castro, I., SantaMaría, G. (1996).- - Triglyceride composition of ewe, cow, and goat milk fat".-J. Am. Oil Chem. Soc., 73: 283-293.

Semporé, G., Bézard, J. (1991).-«Analysis and fractionation of natural source diacylglycerols as urethane derivatives by reversed-phase highperformance liquid chromatography».-J. Am. Oil Chem. Soc., 68: 702-709.

Shekelle, R.B., Missell, L., Oglesby, P. (1985).—«N. Engl. J. Med.".-313: 820.

Shukla, V.K.S., Schiotz Nielsen, W., Batsberg W. (1983)._ «A simple and direct procedure for the evaluation of triglyceride composition of cocoa butters by high-performance liquid chromatography. A comparison with the existing TLC-GC method".Fette Seifen Anstrichm., 85: 274-278.

Singleton, J.A., Pattee, H.W. (1984).-Optimization of parameters for the analysis of triglyceride by reverse phase HPLC using a UV detector".-J. Am. Oil Chem. Soc., 61: 761-766.

Spanos, G.A., Schwartz, S. J., Van Breemen, R.B., Huang, C.H. (1995). - «High-performance liquid chromatography with light-scattering detection and desorption chemical-ionization tandem mass spectrometry of milk fat triacylglycerols".-Lipids, 30: 85-90.

Thompson, G.E. (1988). — «lectrolytes and free fatty acids in milk during involution in the cow».-Res. Vet. Sci., 44: 175-177.

Tsimidou, M., Macrae, R. (1985).—«Reversed-phase chromatography of triglycerides. Theoretical and practical aspects of the influence of injection solvents».-J. Chromatogr. Sci., 23: 155-160.
Wada, S., Koizumi, C., Nonaka, J. (1977).- «Analysis of triglycerides of soybean oil by high performance liquid chromatography in combination with gas liquid chromatography".-Yukagaku, 26: 95-99.

Wada, S., Koizumi, C., Takiguchi, A., Norak, J. (1979).«Triglyceride composition of black cod lipid. II. Possible combinations in triglycerides and their contents".-Bull. Jpn. Soc. Sci. Fish., 45: 615.

Walstra, P., Jenness, R. (1987).-«Química y fisica lactológica".-Zaragoza, Ed. Acribia S.A.

Weber, V.K., Schulte, E., Thier, H.P. (1988).- «HPLC separation of triglycerides in bovine and human milk and GLC analysis of the isolated fractions».-Fat Sci. Technol, 90: 341-344.

Weber, V.K., Schulte, E., Thier, H.P. (1988).— «Triglyceride composition of bovine and human milk».-Fat Sci. Technol., 90: 389-395.

Yang, L.Y., Kuksis, A., Myher, J.J., Steiner, G. (1995)."Origin of triacylglycerol moiety in plasma very low density lipoproteins in the rat: structural studies".-J. Lipid. Res., 36: 125-136.

Yurkowski, M., Brockerhoff, H. (1966).—«Fatty acid distribution of triacylglycerides by deacylation with methylmagnesium bromide".-Biochim. Biophys. Acta, 125: 55-61.

Zegarska, Z. (1988).- - «Seasonal effects on the fatty acids content in milk fat».-Milchwiss, 43: 777-779.

Zegarska, Z., Jaworski, J. (1981).— «Effect of lactation period and seasonal feeding system on quantitative triglyceride composition of milk fat».-Milchwiss, 36: 396-398. 